\title{
A note on the complexity of the bilevel bottleneck assignment problem
}

\author{
Dennis Fischer $^{1}$ D $\cdot$ Komal Muluk $^{1} \cdot$ Gerhard J. Woeginger $^{1}$
}

Received: 24 March 2021 / Revised: 24 March 2021 / Accepted: 14 November 2021 /

Published online: 31 December 2021

(c) The Author(s) 2021

\begin{abstract}
We establish the NP-completeness of the variant of the bilevel assignment problem, where the leader and the follower both have bottleneck objective functions and were the follower behaves according to the optimistic rule. This result settles a problem that has been left open by Klinz \& Gassner [4OR 7:379-394, 2009].
\end{abstract}

Keywords Bilevel programming · Combinatorial optimization · Computational complexity

Mathematics Subject Classification 68Q17 Computational difficulty of problems (lower bounds, completeness, difficulty of approximation, etc.)

\section{Introduction}

A bilevel optimization problem consists of two interleaved optimization problems that are controlled by two non-cooperating decision makers called leader and follower. Both decision makers have a private objective function, a private set of decision variables, and a private set of constraints on their variables. Furthermore there are coupling constraints that connect the decision variables of leader and follower to each other. Both decision makers want to optimize their private objective function. The decision making process is as follows. First the leader makes his decision and fixes the values of his variables, and then the follower reacts by setting his variables. The leader has

Dennis Fischer

fischer@algo.rwth-aachen.de

Komal Muluk

muluk@algo.rwth-aachen.de

Gerhard J. Woeginger

woeginger@algo.rwth-aachen.de

1 Department of Computer Science, RWTH Aachen, Aachen, Germany 
perfect knowledge of the follower's scenario (objective function, variables, and constraints) and also of the follower's behavior. The follower observes the leader's actions, and then optimizes his private objective function subject to the decisions made by the leader (and subject to all the imposed constraints). As the leader's objective function does depend on the follower's decision, the leader must carefully take the follower's reaction into account.

The concept of bilevel optimization goes back to the economic duopoly model of von Stackelberg ( von Stackelberg 1934) from the 1930s. Over the last two decades, bilevel optimization has received enormous interest in the optimization community. We refer the reader to the book by Dempe (2002), to the survey by Brotcorne et al. (2008), and to the annotated bibliographies of Dempe (2003) and Colson et al. (2005) for more information on this area. In a recent paper Gassner and Klinz (2009) consider eight variants of the bilevel assignment problem that arise from the following three binary choices:

- the objective function of the leader is either of sum type or of bottleneck type;

- the objective function of the follower is either of sum type or of bottleneck type;

- the follower either acts according to the optimistic rule or to the pessimistic rule.

The optimistic and the pessimistic rule clarify the situations where the optimal solution for the follower is not unique: While for the follower all optimal solutions yield the same optimal objective value, his choice of the concrete optimal solution influences the objective value of the leader. If the follower acts according to the optimistic rule, he always chooses among his optimal solutions one that is best for the leader. If the follower acts according to the pessimistic rule, he always chooses among his optimal solutions one that is worst for the leader. Gassner and Klinz (2009) establish NPhardness for seven of their eight bilevel assignment variants, and leave the complexity of the eighth variant as an open problem.

The open problem of Gassner \& Klinz. An instance of the bilevel bottleneck assignment problem (BiBAP) is built around a bipartite graph $G=(V, E)$. The edge set $E$ is partitioned into a part $E_{\ell}$ that is controlled by the leader and a part $E_{f}$ that is controlled by the follower. The leader has his own private weight function $w_{\ell}: E \rightarrow \mathbb{R}$ on the edges, and also the follower has a private weight function $w_{f}: E \rightarrow \mathbb{R}$ on the edges.

The optimization process is as follows. First the leader selects a subset $L \subseteq E_{\ell}$, and then the follower selects a subset $F \subseteq E_{f}$ so that $L \cup F$ forms a perfect matching in $G$. The objective of the leader is to minimize his private bottleneck weight $\max \left\{w_{\ell}(e) \mid e \in L \cup F\right\}$, and similarly the objective of the follower is to minimize his private bottleneck weight $\max \left\{w_{f}(e) \mid e \in L \cup F\right\}$. For simplicity of presentation we will assume that in the case of infeasible solutions (where $L \cup F$ does not form a perfect matching), the objective values of leader and follower become infinitely large. As a consequence, the leader will always pick some set $L$ that can be extended to a perfect matching by adding some edges from $E_{f}$, and the follower will always select some set $F$ whose union with $L$ forms a perfect matching. Figure 1 summarizes the resulting bilevel assignment problem, which we call BiBAP. Whenever the minimizer $F$ in (1b) is not uniquely determined, the follower acts according to the optimistic rule and among all possible minimizers selects one that (in combination with the set 


\begin{tabular}{|ll|}
\hline minimize & $\max \left\{w_{\ell}(e) \mid e \in L \cup F\right\}$ \\
& $L \subseteq E_{\ell}$, \\
& and so that $F \subseteq E_{f}$ solves the follower's problem \\
& $\operatorname{minimize}^{*} \max \left\{w_{f}(e) \mid e \in L \cup F\right\}$ \\
& so that $L \cup F$ is a perfect matching
\end{tabular}

Fig. 1 The bilevel bottleneck assignment problem BiBAP studied in this paper

$L$ selected by the leader) yields the best objective value for the leader. In Fig. 1, this optimistic variant of minimization is indicated by the little star in the superscript in the follower's problem.

In this short technical note, we settle the open problem of Gassner and Klinz (2009). We prove that also the eighth and last variant of the bilevel assignment problem is NPcomplete. Though our proof draws some ideas from Gassner and Klinz (2009), it is technically very different and uses a number of new ideas.

\section{The hardness proof}

In this section we present the NP-hardness proof for problem BiBAP. The argument is done through a polynomial time reduction from the NP-hard BALANCED COMPLETE BIPARTITE SUBGRAPH problem; we refer the reader to Garey and Johnson (1979) and to Johnson (1987) for more information on this problem.

\section{Problem: BALANCED COMPLETE BIPARTITE SUBGRAPH (BCBS)}

Instance: A bipartite graph $H=\left(V_{H}, E_{H}\right)$ with bipartition $V_{H}=W_{1} \cup W_{2}$ of the vertex set and with $E_{H} \subseteq W_{1} \times W_{2}$; a positive integer $k$.

Question: Does $H$ contain a complete bipartite subgraph with $k$ vertices on each side of the bipartition?

Now consider an instance of BCBS consisting of the bipartite graph $H=\left(W_{1} \cup\right.$ $\left.W_{2}, E_{H}\right)$ and the integer $k$. Without loss of generality we assume that both sides $W_{1}$ and $W_{2}$ have the same size, and we denote $n=\left|W_{1}\right|=\left|W_{2}\right|$; furthermore we will assume $k \leq n$. We construct the following graph $G=(V, E)$ with $4 n$ vertices and $3 n^{2}-\left|E_{H}\right|+1$ edges:

- For every vertex $v \in W_{1}$ and for every vertex $w \in W_{2}$, the vertex set $V$ contains the corresponding vertices $a(v)$ and $a^{\prime}(w)$.

- Furthermore the vertex set $V$ contains the $2(n-k)$ vertices $b_{1}, \ldots, b_{n-k}$ and $b_{1}^{\prime}, \ldots, b_{n-k}^{\prime}$, and the $2 k$ vertices $c_{1}, \ldots, c_{k}$ and $c_{1}^{\prime}, \ldots, c_{k}^{\prime}$.

- For every $v \in W_{1}$ and $w \in W_{2}$, the edge set $E$ contains the edge $\left\{a(v), a^{\prime}(w)\right\}$ if and only if $\{v, w\} \notin E_{H}$.

- The edge set $E$ connects every vertex $a(v)$ to the $n$ vertices $b_{1}^{\prime}, \ldots, b_{n-k}^{\prime}$ and $c_{1}^{\prime}, \ldots, c_{k}^{\prime}$, and it connects every vertex $a^{\prime}(w)$ to the $n$ vertices $b_{1}, \ldots, b_{n-k}$ and $c_{1}, \ldots, c_{k}$. 
- Finally the edge set $E$ contains the single edge $\left\{c_{k}, c_{k}^{\prime}\right\}$.

Note that the constructed graph $G$ is indeed bipartite, as every edge connects some unprimed vertex to some primed vertex. Next let us specify the edge weights for the leader and for the follower.

- For the leader, the edges $e=\left\{a(v), a^{\prime}(w)\right\}$ all have weight $w_{\ell}(e)=1$, whereas the remaining edges in $E$ have weight 0 .

- For the follower, the $2 n$ edges $e=\left\{a(v), c_{k}^{\prime}\right\}$ and $e^{\prime}=\left\{a^{\prime}(w), c_{k}\right\}$ with $v \in W_{1}$ and $w \in W_{2}$ carry the weight $w_{f}(e)=w_{f}\left(e^{\prime}\right)=1$. All other edges in $E$ have weight 0 for the follower.

Finally, we specify the edge sets $E_{\ell}$ and $E_{f}$ controlled by leader and follower.

- The set $E_{\ell}$ consists of the edges $\left\{a(v), b_{j}^{\prime}\right\}$ and $\left\{a^{\prime}(w), b_{j}\right\}$ with $v \in W_{1}, w \in W_{2}$ and $1 \leq j \leq n-k$.

- The set $E_{f}$ contains all the remaining edges: the edges $\left\{a(v), a^{\prime}(w)\right\}$, the edges $\left\{a(v), c_{i}^{\prime}\right\}$ and $\left\{a^{\prime}(w), c_{i}\right\}$, and the single edge $\left\{c_{k}, c_{k}^{\prime}\right\}$.

This completes the construction of the BiBAP instance. Since all edge weights $w_{\ell}$ and $w_{f}$ only take the values 0 and 1 , the possible objective values for leader and follower are 0 and 1 . We next state some simple observations on the behavior of the leader and the follower. Note that the matching $L \subseteq E_{\ell}$ selected by the leader contains at most $2(n-k)$ edges, since every edge in $E_{\ell}$ is incident to one of the $2(n-k)$ vertices $b_{1}, \ldots, b_{n-k}$ and $b_{1}^{\prime}, \ldots, b_{n-k}^{\prime}$,

Lemma 1 Let $L \subseteq E_{\ell}$ be an arbitrary matching chosen by the leader.

(i) If $|L|<2(n-k)$, then no reaction $F \subseteq E_{f}$ of the follower can yield a perfect matching $L \cup F$ for the graph $G$. Hence in this case, the objective values of the leader and of the follower are infinite.

(ii) If $|L|=2(n-k)$, then there exists a reaction $F \subseteq E_{f}$ of the follower that yields a perfect matching $L \cup F$ in graph $G$. Hence in this case, the objective values of the leader and of the follower are finite.

Proof For (i), observe that the $2(n-k)$ vertices $b_{1}, \ldots, b_{n-k}$ and $b_{1}^{\prime}, \ldots, b_{n-k}^{\prime}$ are pairwise non-adjacent and are only incident to edges controlled by the leader. If $|L|<$ $2(n-k)$, then one of these $2(n-k)$ vertices will not be saturated by $L \cup F$.

For (ii), observe that every matching $L \subseteq E_{\ell}$ with $|L|=2(n-k)$ saturates the $2(n-k)$ vertices $b_{1}, \ldots, b_{n-k}$ and $b_{1}^{\prime}, \ldots, b_{n-k}^{\prime}$ together with $n-k$ of the $a$-vertices and $n-k$ of the $a^{\prime}$-vertices. The unsaturated vertices may be divided into two groups: The first group consists of the $k$ unsaturated $a$-vertices together with $c_{1}^{\prime}, \ldots, c_{k}^{\prime}$, and the second group consists of the $k$ unsaturated $a^{\prime}$-vertices together with $c_{1}, \ldots, c_{k}$. As either group induces a balanced complete bipartite subgraph in $G$ and as all edges in these two induced subgraph belong to set $E_{f}$, the follower easily extends $L$ to a perfect matching $L \cup F$ for $G$.

Lemma 2 Let $L \subseteq E_{\ell}$ with $|L|=2(n-k)$ be some matching chosen by the leader, and let $F \subseteq E_{f}$ be the optimal reaction of the follower to $L$.

(i) If $F$ does not contain any of the edges $\left\{a(v), a^{\prime}(w)\right\}$ with $v \in W_{1}$ and $w \in W_{2}$, then the leader has objective value 0 and the follower has objective value 1 . 
(ii) If $F$ contains one of the edges $\left\{a(v), a^{\prime}(w)\right\}$ with $v \in W_{1}$ and $w \in W_{2}$, then the leader has objective value 1 and the follower has objective value 0 .

Proof The only edges with non-zero weight for the leader are the edges $\left\{a(v), a^{\prime}(w)\right\}$, and all these edges are controlled by the follower. Hence the leader has objective value 0 in (i) and objective value 1 in (ii).

Let $U$ denote the set of vertices $a(v)$ with $v \in W_{1}$ that are not saturated by $L$, and let $U^{\prime}$ denote the set of vertices $a^{\prime}(w)$ with $w \in W_{2}$ that are not saturated by $L$. Note that $|U|=\left|U^{\prime}\right|=k$. In (i) the follower must saturate the vertices in $U$ by matching them to $c_{1}^{\prime}, \ldots, c_{k}^{\prime}$. As the edge to vertex $c_{k}^{\prime}$ has weight 1 for the follower, his objective value is 1 . In (ii) the follower saturates one of the vertices in $U$ and one of the vertices in $U^{\prime}$ by picking an edge $\left\{a(v), a^{\prime}(w)\right\}$ for $F$. The remaining $k-1$ vertices in $U$ can be matched to $c_{1}^{\prime}, \ldots, c_{k-1}^{\prime}$, the remaining $k-1$ vertices in $U^{\prime}$ can be matched to $c_{1}, \ldots, c_{k-1}$, and finally the two vertices $c_{k}$ and $c_{k}^{\prime}$ can be matched to each other at cost 0 . This yields an objective value of 0 for the follower.

Lemma 3 In the BiBAP instance the leader can reach an objective value of 0 , if and only if the bipartite graph $H=\left(W_{1} \cup W_{2}, E_{H}\right)$ in the BCBS instance possesses a complete bipartite subgraph with $k$ vertices on each side of the bipartition.

Proof (If) First assume that there exist subsets $X_{1} \subseteq W_{1}$ and $X_{2} \subseteq W_{2}$ with $\left|X_{1}\right|=$ $\left|X_{2}\right|=k$ so that $X_{1} \cup X_{2}$ induces a complete bipartite subgraph in $H$. Then the leader picks for his set $L$ a perfect matching between $b_{1}^{\prime}, \ldots, b_{n-k}^{\prime}$ and the vertices $a(v)$ with $v \in W_{1}-X_{1}$ together with a perfect matching between $b_{1}, \ldots, b_{n-k}$ and the vertices $a^{\prime}(w)$ with $w \in W_{2}-X_{2}$. Since $X_{1} \cup X_{2}$ induces a complete bipartite subgraph in graph $H$, the unsaturated $a$-vertices and $a^{\prime}$-vertices induce an independent set in graph $G$. Now Lemma 2.(i) completes the argument.

(Only if) Next assume that graph $H$ does not contain a complete bipartite subgraph with $k$ vertices on each side of the bipartition. Let $Y_{1} \subseteq W_{1}$ denote the vertices $v \in W_{1}$ for which $L$ does not saturate $a(v)$, and let $Y_{2} \subseteq W_{2}$ denote the vertices $w \in W_{2}$ for which $L$ does not saturate $a^{\prime}(w)$. Then $\left|Y_{1}\right|=\left|Y_{2}\right|=k$, and by our assumption the subgraph of $H$ induced by $Y_{1} \cup Y_{2}$ can not be complete. Hence there exists at least one edge $e=\left\{a(v), a^{\prime}(w)\right\}$ in $G$, so that the matching $L$ does neither saturate $a(v)$ nor $a^{\prime}(w)$. Lemma 2 yields the small objective value 0 for the follower in case he puts such an edge $e$ into his set $F$, and a large objective value 1 for the cases where he does not use any such edge. Hence the follower will choose such an edge $e$ for $F$, and will thereby yield an objective value of 1 for the leader.

Lemma 3 establishes the correctness of our reduction: The optimal objective value of the leader in the constructed BiBAP instance tells us the answer to the BCBS instance. Since the edge weights introduced in the reduction are polynomially bounded in the instance size, the reduction actually yields NP-hardness in the strong sense. Finally, we note that the decision version of problem BiBAP is clearly contained in NP. All in all, we formulate the following summarizing theorem.

Theorem 4 The decision version of the bilevel assignment problem with a bottleneck objective function where leader and follower have bottleneck objective functions and 
where the follower behaves according to the optimistic rule is NP-complete in the strong sense.

The polynomial time reduction from BCBS to BiBAP is simple and highly structured. In the constructed instance of BiBAP, the follower has so little decision power that behavior according to the optimistic rule and behavior according to the pessimistic rule yield the same outcome. Furthermore, it can be verified that the behavior of leader and follower also does not change, if one or both of them switch from bottleneck objective function to sum objective function. Hence, our construction yields as a byproduct a unified alternative proof for the seven hardness results that have been derived in Gassner and Klinz (2009). Finally, we remark that our reduction also implies the inapproximability of problem BiBAP: Unless $P=N P$, there is no polynomial time approximation algorithm for BiBAP that approximates the optimal objective value of the leader with some constant worst case ratio. We omit the straightforward details.

Funding Open Access funding enabled and organized by Projekt DEAL. The research leading to these results received funding from Deutsche Forschungsgemeinschaft (DFG) under the grant DFG RTG 2236 "UnRAVeL".

\section{Declarations}

Conflict of interest The authors certify that they have no affiliations with or involvement in any organization or entity with any financial interest or non-financial interest in the subject matter or materials discussed in this manuscript.

Open Access This article is licensed under a Creative Commons Attribution 4.0 International License, which permits use, sharing, adaptation, distribution and reproduction in any medium or format, as long as you give appropriate credit to the original author(s) and the source, provide a link to the Creative Commons licence, and indicate if changes were made. The images or other third party material in this article are included in the article's Creative Commons licence, unless indicated otherwise in a credit line to the material. If material is not included in the article's Creative Commons licence and your intended use is not permitted by statutory regulation or exceeds the permitted use, you will need to obtain permission directly from the copyright holder. To view a copy of this licence, visit http://creativecommons.org/licenses/by/4.0/.

\section{References}

Brotcorne L, Marcotte P, Savard G (2008) Bilevel programming: the Montreal school. INFOR: Inf Syst Op Res 46:231-246

Colson B, Marcotte P, Savard G (2005). Bilevel programming: a survey. 4OR 3, 87-107

Dempe S (2002) Found Bilevel Progr. Kluwer Academic Publishers, Dordrecht

Dempe S (2003) Annotated bibliography on bilevel programming and mathematical programs with equilibrium constraints. Optim 52:333-359

Garey MR, Johnson DS (1979) Computers and intractability: a guide to the theory of NP-completeness. Freeman, San Francisco

Gassner E, Klinz B (2009). The computational complexity of bilevel assignment problems. 4OR 7, 379-394 Johnson DS (1987) The NP-completeness column: an ongoing guide. J Algorithms 8:438-448

von Stackelberg H (1934). Marktform und Gleichgewicht. Springer, Berlin [English translation: The theory of market economy, Oxford University Press, 1952]

Publisher's Note Springer Nature remains neutral with regard to jurisdictional claims in published maps and institutional affiliations. 